\title{
Überlegungen zu Methoden und Modellen der prozessualen Übersetzungsforschung
}

\author{
Fairuz Ezzat Shaker Gawargi \\ Oberassistentin an der Abteilung für Germanistik \\ der Sprachenfakultät (Al-Alsun) der Minia Universität \\ fairuzgawargi@yahoo.de
}

\begin{abstract}
Abstrakt
Der vorliegende Beitrag fokussiert einen modernen, einen sich rasant entwickelnden Forschungsaspekt der Übersetzungswissenschaft, und zwar die prozessuale Übersetzungsforschung. Die wissenschaftlichen Untersuchungen interessieren sich nicht mehr für die Hypothesenbildung über den Ablauf des Übersetzungsprozesses anhand des Vergleichs zwischen dem AT und dem ZT. Stattdessen werden Methoden und Verfahren der Kognitiven Wissenschaft bei Versuchspersonen während des Übersetzens angewandt, um die ablaufenden mentalen Vorgänge zu erhellen und dementsprechend empirisch begründete Modelle des Übersetzungsprozesses zu erstellen. Der Beitrag setzt sich zum Ziel, diejenigen Methoden und Verfahren $\mathrm{zu}$ erklären, derer sich die prozessuale Übersetzungsforschung bedient. Darüber hinaus stellt diese Forschungsarbeit die verschiedenen, von den Vertretern der prozessorientierten Forschung erstellten Modelle des Übersetzungsprozesses dar. Die vorliegende Forschungsarbeit gliedert sich in eine Einleitung, drei Hauptteile, eine Zusammenfassung und ein Literaturverzeichnis. Der Beitrag bietet zunächst einen Überblick über den Gegenstand und die Zielsetzung der Untersuchung. Der erste Teil führt dann den Leser in den Forschungsbereich der prozessorientierten Übersetzungsforschung ein. Im zweiten Teil werden die Methoden und Verfahren der prozessualen Forschung zusammengefasst. Der dritte Teil stellt ausführlich die verschiedenen Übersetzungsprozessmodelle dar. Eine komprimierte Zusammenfassung und ein Ausblick sowie das Literaturverzeichnis schließen die Forschungsarbeit ab.
\end{abstract}

Schlüsselwörter: prozessorientierte Übersetzungswissenschaft - Kognitive Linguistik - Übersetzungsprobleme und -strategien - introspektive Verfahren

\section{Abkürzungsverzeichnis}
AT: Ausgangstext
LD Lautes Denken
KI: Künstliche Intelligenz
ZT: $\quad$ Zieltext 


\section{Einleitung}

Die Fähigkeit zu sprechen ist eine hohe kognitive Leistung, die die menschlichen Lebewesen charakterisiert. Die Sprache hilft dem Menschen, sich in der Welt zu orientieren sowie Erfahrungen zu machen und diese weiterzuvermitteln. Eine Form der sprachlichen Kommunikation stellt das Übersetzen dar, das heute einen unabdingbaren Bestandteil der globalisierten Welt bildet. In den verschiedenen Lebensbereichen besteht immer das Bedürfnis, Texte anderer Kulturen - und somit Sprachen - in die eigene Sprache zu übernehmen, und umgekehrt.

Das Übersetzen ist seit der Antike unter theologischem, philosophischem, ästhetischem, psychologischem und anthropologischem Aspekt Gegenstand der theoretischen und methodischen Forschung. In der modernen Auffassung hat sich die Übersetzungswissenschaft als eine empirische Interdisziplin etabliert, an deren Entwicklung verschiedene Wissenschaften Anteil haben wie: die Semiotik, die Psychologie und die Kognitionswissenschaft (vgl. Wills, 1988: 2f.).

Ein junger Forschungsansatz, dessen Methoden und Theorien die Übersetzungsforschung maßgebend beeinflussen, ist die Kognitiven Linguistik, die Aufgabe hat, die Mechanismen der sprachlichen Kommunikation und ihre Prinzipien systematisch zu beschreiben. Die übersetzerischen Fragestellungen werden hauptsächlich aus zwei Perspektiven behandelt: von den textbezogenen oder von den verhaltensbezogenen Ansätzen. Bei den textbezogenen Ansätzen handelt es sich um Theorienbildung anhand des Vergleichs zwischen dem AT und dem ZT. Solche Ansätze haben in der Übersetzungswissenschaft eine lange Tradition. Erst nach der kognitiven Wende sowohl in der Psychologie als auch in der Linguistik beginnen die verhaltensbezogenen Ansätze sich durchzusetzen. Dabei unterscheidet man weiter zwischen eher bedingungsanalytischen Vorgehensweisen, die sich auf die Bestimmung der am Übersetzungsvorgang beteiligten psychologischen Bedingungen konzentrieren, und eher prozessanalytischen Vorgehensweisen, die noch die Erforschung der inneren psychischen Zustände miteinbeziehen, die die psychologischen Bedingungen bewirken (vgl. Grabowski, 1999: 98ff.). 
Mein Beitrag steht im Rahmen des letzten Forschungszweigs, der sog. prozessorientierten Übersetzungswissenschaft bzw. die prozessuale Übersetzungsforschung, deren Entwicklung in den 1980er Jahren begonnen hat. Dabei steht im Vordergrund der wissenschaftlichen Untersuchung nicht mehr das Produkt des Übersetzens, sondern der Prozess des Übersetzens, seine Modellierung und seine Didaktisierung. Der Beitrag stellt die verschiedenen Versuche der prozessual arbeitenden Übersetzungswissenschaftler dar, den Übersetzungsprozess zu modellieren. Dadurch kann der Übersetzer den Einzelheiten seiner Aktivität bewusst sein.

\section{Die prozessuale Übersetzungsforschung: Geschichte und Entwicklung}

Die Übersetzungswissenschaft hat im Allgemeinen eine sehr lange alte Geschichte, die bis zum Altertum zurückreicht. Die Entwicklung der Übersetzungswissenschaft über mehrere Jahrtausende hinweg geschah nicht geradlinig, sondern ist durch das Nebeneinander und Gegeneinander verschiedener übersetzungstheoretischer und methodischer Standpunkte und Prinzipien gekennzeichnet (vgl. Stolze, 2008: 9).

Innerhalb der Übersetzungswissenschaft ist die Übersetzungsprozessforschung ein junger Forschungsbereich, mit dessen Beschäftigung in den 80er Jahren des 20. Jahrhunderts angefangen wurde. Bis zu diesem Zeitpunkt wurden nur die Übersetzungsprodukte modelliert. Bei der Beschreibung der Übersetzungsprozesse handelt es sich „um theoretische Modellierungen aus linguistischer, semiotischer, kommunikationstheoretischer, text- oder literaturwissenschaftlicher Sicht, nicht aber um den Versuch, konkrete Übersetzungsereignisse empirisch zu beschreiben“. Erst Mitte der 80er Jahre des 20. Jahrhunderts erschienen mehrere prozessorientierte Studien zum Übersetzungsprozess, die empirisch begründet waren. Hierzu gehören: Krings (1986), Gerloff (1988) und Lörscher (1991). Zu erwähnen ist, dass diese Studien unabhängig voneinander entstanden sind und mithilfe der introspektiven Verfahren verbale Daten über die mental ablaufenden Prozesse während des Übersetzens erhoben haben (vgl. Krings, 2005: 342f.).

Zur Entwicklung der prozessorientierten Übersetzungswissenschaft haben die Forschungsergebnisse der Kognitiven Wissenschaft beigetragen. Innerhalb der 
Psychologie unterscheidet man zwischen dem behavioristischen und dem kognitivistischen Paradigma. Die behavioristische Psychologie setzte sich über viele Jahrtausende hinweg mit dem Anspruch durch, die Funktionsweise des menschlichen Geistes zu untersuchen, wobei nur das beobachtbare Verhalten des Menschen analysiert wurde. In den 60er und 70er Jahren des 20. Jahrhunderts fand ein Paradigmenwechsel statt, was heute als kognitive Wende bezeichnet wird. Im Unterschied zum Behaviorismus stehen im Zentrum der kognitivistischen Sichtweise, die nach Neisser Kognitive Psychologie genannt wurde, die Denk- und Verstehensprozesse des Lernenden. Der Kognitivismus konzentriert sich auf das Geheimnis der sog. Blackbox des menschlichen Gehirns, d.h. auf die beim Lernen intern im Gehirn ablaufenden Prozesse (vgl. Orban, 2008: 18f.).

Die kognitive Wende übertrug Chomsky in der Linguistik, indem er die generative Linguistik als ein Teilgebiet der kognitiven Psychologie betrachtete. Chomskys Sprachtheorie gab den Anstoß zur Entwicklung kognitivistischer Theorien in der Linguistik (vgl. Rickheit / Weiss / Eikmeyer, 2010: 10f.).

Einen wichtigen Einfluss auf Linguistik und Psychologie in Bezug auf die Darstellung mentaler Wissensstrukturen und die Modellierung kognitiver Prozesse haben die Programme und die Simulationsstudien der Computerwissenschaft genommen. Mitte der 70er Jahre wurde dank der Annäherung von KI-Forschung und Psychologie die sog. Kognitive Wissenschaft entwickelt, die Erkenntnisse aus der Psychologie, der Computerwissenschaft, der Linguistik, der Philosophie und der Neurowissenschaft integriert. Die jeweiligen Disziplinen fokussieren bei ihren Untersuchungen bestimmte Aspekte der Kognition. Demgemäß setzt sich die interdisziplinäre Kognitive Wissenschaft zum Ziel, durch die Kooperation der Fachbereiche menschliche Kognition in ihrer Komplexität umfassend zu erforschen (vgl. Schwarz, 2008: 17ff.).

Die Kognitive Linguistik versteht sich einerseits als eine Teildisziplin der Sprachwissenschaft und andererseits als eine Teildisziplin der Kognitiven Wissenschaft. Sie repräsentiert einen Forschungsansatz, der Sprache als ein geistiges Kenntnis- und Prozessorsystem sieht. Ausgehend davon hat die Kognitive Linguistik die Aufgabe, die mentalen Sprachstrukturen und -prozesse zu beschreiben und die 
kognitive Realität von Sprache zu modellieren. Es geht um die Frage, wie sprachliches Wissen mental repräsentiert und kognitiv verarbeitet wird (vgl. Felix / Habel / Rickheit, 1994: 1f.).

Die Kognitive Linguistik betont, dass Erkenntnisse des rein syntaktischen Ansatzes nicht ausreichend sind, um die menschlichen Sprachfähigkeit zu erforschen. Sie bezieht die lange vernachlässigte Komponente der Semantik mit ein und versucht, Aspekte der prozeduralen Kompetenz $\mathrm{zu}$ erfassen, indem die Schnittstellen zwischen dem sprachlichen System und den anderen kognitivem Subsystemen bei der Sprachverarbeitung analysiert werden. „,Ein fundamentales Anliegen der Kognitiven Linguistik ist es, strukturelle und prozedurale Aspekte sprachlichen Wissens enger aufeinander zu beziehen" (Schwarz, 2008: 46).

Die Grundlage der modernen Kognitiven Linguistik bilden die Kenntnisse der psycho-, neuro- und computerlinguistischen Forschungsperspektiven:

Die gegenwärtige Psycholinguistik zielt darauf $a b$, die medialen und mentalen Charakteristika der sprachlichen Äußerungen zu erforschen, um Erkenntnisse darüber zu erhalten, wie die Menschen mit ihrer Sprache umgehen. Ihr Forschungsspektrum umfasst drei große Teilbereiche, nämlich: das Sprachwissen, die Sprachprozesse und den Spracherwerb (vgl. Rickheit / Sichelschmidt / Strohner, 2007: 15).

Die Neurolinguistik integriert Ergebnisse aus der Linguistik und den Neurowissenschaften, um neurokognitive Modelle zu bilden. Die neurolinguistische Forschung richtet sich auf den Zusammenhang von Sprache und Kognition und deren neurobiologischen Korrelaten (vgl. Rickheit / Weiss / Eikmeyer 2010: 100ff.).

Die Computerlinguistik ist ein interdisziplinäres Fachgebiet, das eine Schnittstelle zwischen der Sprachwissenschaft und der Informatik darstellt. Sie wendet die mit dem Computer verbundenen Verfahren und Methoden an, um die sprachlichen Strukturen und die Sprachverarbeitungsprozesse zu beschreiben. Ihr Hauptziel ist die Untersuchung, wie natürliche Sprache durch formale Modellierung, algorithmische Verfahren der Informatik und statistische Verfahren automatisch verarbeitet und interpretiert werden kann (vgl. http://www.uniheidelberg.de/studium/ interesse/faecher/computerling_ba.html, am 06.12.2016). 
Neben den klassischen computerlinguistischen symbolverarbeitende Modelle zur Verfügung, die die linguistischen Gegenstände als Zeichenketten betrachten, entspricht die moderne konnektionistische Modellierung den Ansprüchen der Kognitiven Linguistik (vgl. Rickheit / Weiss / Eikmeyer 2010: 193ff.).

Inspiriert wurde die Untersuchung der Übersetzungsfragestellungen aus prozessualer Sicht von den Ergebnissen der Kognitiven Linguistik mit ihren psycholinguistischen, neurolinguistischen und computerlinguistischen Perspektiven. Der psycholinguistische Beitrag stammt von den amerikanischen Psychologen K. Anders Ericsson und Herbert A. Simon mit ihrem 1984 erschienenen Werk „Protocol Analysis: Verbal Reports as Data“, in dem sie die methodologischen Grundlagen für die Gewinnung verbaler Daten mithilfe der introspektiven Methoden erklären. Weiter belebt wurde die Übersetzungsprozessforschung durch die Entwicklung einer Schreib-Logging-Software, die alle Tastenanschläge und Mausklicks des Übersetzers und die Zeitintervalle zwischen diesen erfassen kann. Andererseits haben es die in den Neurowissenschaften entwickelten bildgebenden Verfahren der Übersetzungsprozessforschung ermöglicht, Aufschluss über die an dem Übersetzungsprozesses beteiligten Gehirnregionen zu bekommen (vgl. Göpferich, 2008: 4ff.).

Die gegenwärtige Übersetzungsprozessforschung ist dadurch gekennzeichnet, dass sie die Ergebnisse ihrer qualitativen Auswertungsmethoden durch Anwendung quantitativer statistischer Verfahren ergänzt, wie z: B. Fragebögen und Eye-Tracking (vgl. Göpferich, 2008: 6f.).

\section{Methoden und Verfahren}

Die prozessorientierte Übersetzungsforschung verzichtet auf den Vergleich zwischen dem AT und dem ZT. Stattdessen fokussiert sie die Denkphasen des Übersetzungsprozesses und versucht, sie zu modellieren. Ausgehend davon werden Methoden und Verfahren aus den Bereichen der Kognitiven Wissenschaft entnommen, um Daten über die während des Übersetzens ablaufenden Prozesse zu erheben. Die nächsten Unterteile versuchen, die einzelnen Methoden sowie Datenerhebungs- und -auswertungsverfahren zu diskutieren. Da sie facettenreich 
sind, werden sie in Gruppen eingeteilt, wobei die Hauptgedanken von Krings (1986: 63f.), Hansen (2006: 53ff.) und Göpferich (2008: 16ff.) wiedergegeben werden.

\subsection{Introspektive Verfahren}

Die introspektiven Verfahren wurden von Wilhelm Wundt Ende des 19. Jahrhunderts in die deutsche Experimentalpsychologie eingeführt und erlebten mit der kognitiven Wende einen Aufschwung. Der Begriff „Introspektion“ wird in drei unterschiedlichen Bedeutungen verwendet:

1. im Sinne von Selbstbeobachtung bestimmter Bewusstseinsinhalte;

2. die Retrospektion, die auf Introspektion im weiteren Sinne hinweist und als Oberbegriff für alle Verfahren gebraucht wird, bei denen die Versuchspersonen alle Gedanken und Empfindungen bei der Lösung der Aufgabe verbalisieren.

3. Introspektion im engeren Sinne, die als Komplementärbegriff zur Introspektion gilt und bei der die verbalen Daten während der Lösung der Aufgabe erfasst werden.

Bevor die einzelnen Methoden der introspektiven Verfahren ausführlich diskutiert werden, ist die Bedeutung der introspektiven Daten für die Übersetzungswissenschaft trotz des Zweifels an deren Aussagekraft zu betonen. House macht das deutlich (2000: 152; nach Göpferich, 2008: 21f.):

Suffice it to say here that despite the fact that there remain basically undispelled doubts about the status of introspective data in translation process research, the pedagogical potential of this research is considerable for at least the following four reasons: 1 . because of its attempt to go beyond visible translation practices and surface linguistic realization trying to investigate underlying cognitive processes responsible for the creation of surface forms, 2. Because it leads to descriptive statements instead of normative ones, 3. Because it proceeds in an empiricallyinductive instead of a theoretically-deductive way, and last but not least, 4. Because this type of research is oriented towards the practice translation.

\section{Methode des lauten Denken (LD-Methode)}

Die Methode des lauten Denkens, auch Protokollanalyse genannt, ist zur Untersuchung der schriftlichen Sprachproduktion geeignet. Die Versuchspersonen erhalten eine schriftliche Aufgabe. Sie sollen alle Gedanken verbalisieren, die ihnen beim Schreiben durch den Kopf gehen. Ihre Äußerungen werden auf Tonträgern 
aufgenommen, $\mathrm{zu}$ LD-Protokollen transkribiert und unter verschiedenen Gesichtspunkten analysiert.

Als eine Methode zur Untersuchung die ablaufenden mentalen Prozesse hat das laute Denken einige Vorteile, und zwar:

- Man bekommt mithilfe der LD-Methode umfangreichere und konkretere Informationen $\mathrm{zu}$ den mentalen Prozessen als bei allen anderen bis dahin angewandten Verfahren, da sie uns Aufschluss über die komplexen Problemlösungsprozesse, die Gedankengänge und die kreativen Strategien geben kann.

- Die LD-Methode hilft bei der Hypothesenbildung, weil durch diese Methode Daten in unstrukturierter Form und unabhängig von Modellvorstellungen erhoben werden.

- Aufgrund der LD-Daten erhält man Kenntnis über die subjektiven Theorien der Versuchspersonen bei der Lösung der Aufgaben. Diese subjektiven Theorien sind für die Entwicklung der übersetzerischen Kompetenz und die Bewertung der angewandten Übersetzungsstrategien von besonderer Bedeutung.

Auf der anderen Seite werden gegen die Validität von LD-Daten einige Überlegungen vorgebracht:

- Viele zweifeln die Konsistenz der verbalisierten Daten mit den tatsächlichen Bewusstseinsinhalten an aus dem Grund, dass die von einem geäußerten Bewegründe nicht unbedingt die tatsächliche Kognition widerspiegeln, die die Handlungen steuert.

- Ein weiterer Nachteil liegt in der Interferenz zwischen der Verbalisierung und dem eigentlichen Problemlösungsprozess. Das Argument dafür ist, dass das laute Denken ein unnatürlicher Vorgang ist.

- Es wird behauptet, dass die LD-Daten nur bewusste Prozesse widerspiegeln.

Dass das Nutzen von LD-Daten in Frage gestellt wird, kritisiert Krings, indem er die Verbalisierungen mit einem U-Boot vergleicht, das dann und wann auftaucht und nicht permanent verfolgt werden kann. Wir kennen darum seinen genauen Weg nicht, können aber seinen Kurs genau rekonstruieren. 
Um diese Nachteile bei der Durchführung von LD-Studien zu vermeiden und valide Daten zu bekommen, empfiehlt Göpferich, folgende Punkte zu beachten:

1. Vor Versuchsbeginn soll der Versuchsleiter präzise Daten über die Versuchspersonen haben. Dazu gehören: Ausbildung; Motivation, das Niveau der Sprachenbeherrschung usw.

2. Den Versuchspersonen soll die Aufgabe klar sein.

3. Eine Aufwärmphase ist für die Versuchspersonen wichtig, um sich mit den verwendeten Programmen vertraut zu machen.

4. Die Versuchspersonen sollen nicht aufgefordert werden, ihr Verhalten während der Studie zu begründen.

5. Der Versuchsleiter soll nicht im Blickfeld der Versuchspersonen sein.

6. Von Bedeutung ist auch, dass die Studien textsortenspezifisch durchgeführt werden.

7. Es ist besser, dass die Versuchspersonen den Zweck der Studie nicht kennen, damit sie ihre Gedanken spontan äußern.

\section{Retrospektive Befragungen}

Die retrospektiven Befragungen können als Ergänzung zur Erhebung von LD-Daten dienen und Aufschluss über die mentalen Prozesse geben, die während der Sprechpausen beim Übersetzen vorkommen. Die Versuchspersonen können außerdem die retrospektiven Befragungen verwenden, um bestimmte Begrifflichkeiten und Bezeichnungen abzuklären und dabei Missverständnisse zu vermeiden.

\section{Integrated Problem and Decision Reporting}

Integrated Problem and Decision Reporting ist eine spezielle Form der retrospektiven Kommentierungen, bei der die Versuchspersonen ihre Übersetzungen in einer beliebigen Form kommentieren sowie alle ihre Übersetzungsprobleme und Lösungsfindungsstrategien beschreiben und begründen sollen.

Diese Methode fokussiert die Bedürfnisse der Studenten und kann somit als ein didaktisches Mittel genutzt werden. Sie hilft dem Lehrer dabei, die Übersetzungsleistungen der Studenten zu interpretieren, die Entwicklung ihrer 
übersetzerischen Kompetenz $\mathrm{zu}$ bewerten und die didaktischen Methoden zu überprüfen.

\section{Fragebögen}

Die Wissenschaftler bedienen sich Fragebögen, um auf wichtige spezifische Aspekte des Übersetzungsprozesses einzugehen. Anstatt von formlosen Kommentaren werden Daten in Formularen mit entsprechenden Kategorien erhoben.

\section{Dialogprotokolle}

Bei den Dialogprotokollen geht es um die Aufzeichnungen der Verbalisierungen von zwei oder mehreren Versuchspersonen, die gemeinsam einen Text übersetzen und dabei alle ihre Gedanken laut äußern. Im Vergleich zur LD-Methode haben die Dialogprotokolle den Vorteil, dass sie weniger künstlich sind und den Versuchspersonen erlauben, unbefangen zu reden.

Im Gegensatz dazu haben die aus den Dialogprotokollen gewonnenen Daten geringere Konsistenz mit den eigentlichen mentalen Prozessen als die LD-Daten. Der Grund dafür liegt darin, dass die Versuchspersonen nicht mehr ungefiltert aussprechen, sondern die Gesprächspartner beachten und ihre Lösungen begründen. Außerdem können die Versuchspersonen beim Denken gestört und unterbrochen werden. Den Dialogprotokollen fehlt noch die ökologische Validität, da die TeamÜbersetzungen in der Praxis kaum vorkommen. Trotzdem können die Dialogprotokolle als ein wichtiges didaktisches Mittel betrachtet werden, durch das die an den Dialogen beteiligten Personen die Lösungsstrategien voneinander kennenlernen können.

\subsection{Schreib-Logging und Pausenanalysen}

Die Erforschung des Schreibprozesses beim Übersetzen kann dazu beitragen, die übersetzerische mentale Blackbox des Übersetzers zu erhellen. Neben den Papieren und Stiften werden heute zur Erfassung des Schreibprozesses am Computer spezielle Schreib-Logging-Programme entwickelt. Während die Versuchsperson am Computer arbeitet, zeichnen diese Programme im Hintergrund alle Aktivitäten der Tastenanschläge und Mausklicks auf. Die Log-Daten geben dann Aufschluss über 
den Ablauf des Schreibprozesses und die gesamten Zwischenversionen. Ein in der Übersetzungswissenschaft am häufigsten verwendetes Programm ist Translog, das zum ersten Mal an der Copenhagen Business School entwickelt wurde und bis jetzt revidiert und weiterentwickelt wird.

Ein Nachteil der Schreib-Logging-Programme ist, dass sie die Aktionen außerhalb des Programms nicht aufzeichnen können, wie z.B. das Recherchieren im Internet und die Notizen auf Zetteln. Um diesen Nachteil zu vermeiden, wird empfohlen, dass ein Schreib-Logging-Programm zusammen mit einer ScreenRecording-Software und einer Videokamera eingesetzt wird.

\subsection{Bildgebende Verfahren}

Unter bildgebenden Verfahren versteht man „die Gesamtheit der apparativen Verfahren, mit denen beispielsweise medizinische Befunde oder physikalische und chemische Phänomene visualisiert werden" (Bildgebendes Verfahren, 2006: 1; nach Göpferich, 2008: 63). Die bildgebenden Verfahren machen z. B. die Gehirnaktivitäten lokalisierbar und sichtbar. $\mathrm{Zu}$ ihnen gehören die Funktionelle Magnetresonanztomografie und die Positronenemissionstomografie.

\section{Modelle der prozessualen Übersetzungsforschung}

Anhand der mittels der dargestellten Methoden und Verfahren erhobenen Daten versuchen die Vertreter der prozessualen Übersetzungsforschung, Modelle des Übersetzungsprozesses zu erstellen. Die folgenden drei Unterteile stellen die Merkmale der verschiedenen Modelle anhand der Ausführungen von Krings (1986), Hönig (1997) und Göpferich (2008) dar.

\subsection{Krings' Modell}

Krings hat ein vorläufiges Modell des Übersetzungsprozesses aus den Daten seiner LD-Studien entwickelt, bei denen er den Übersetzungsprozess bei fortgeschrittenen Französischlernern mit deutscher Muttersprache untersuchte. Der Schwerpunkt liegt auf den Übersetzungsproblemen und den zu ihrer Lösung angewandten Strategien. Für Krings sind die Übersetzungsstrategien „potenziell bewußte Pläne eines 
Übersetzers zur Lösung konkreter Übersetzungsprobleme im Rahmen einer konkreten Übersetzungsaufgabe “(Krings, 1986: 175).

Laut dem Modell von Krings werden keine Strategien angewandt und der Übersetzungsprozess verläuft automatisiert $a b$, wenn der Übersetzer eine ausgangssprachliche Einheit direkt in eine zielsprachliche überträgt. Strategien kommen erst zur Anwendung, wenn er Probleme hat, ein zielsprachliches Äquivalent automatisch zu finden. Nach Auffassung von Krings können die Übersetzungsstrategien auf zwei Ebenen auftreten: auf eine Makro- und eine Mikroebene.

Die Makrostrategien beschreibt Krings anhand eines Dreiphasenmodells. Drei Phasen werden unterschieden:

1. die vorbereitende Phase (Vorlauf), die der psychischen Entlastung dient;

2. die eigentliche Übersetzungsphase (Hauptlauf);

3. die nachbereitende Phase (Nachlauf).

Auf der Mikroebene differenziert Krings fünf Kategorien der Strategien:

- Rezeptionsstrategien, die sich auf Verstehensprobleme beziehen;

- Äquivalentauffindungsstrategien, zu denen die Äquivalentassoziationen gehören;

- Evaluationsstrategien, die eine Monitor- und Korrekturfunktion haben;

- Entscheidungsstrategien, die angewandt werden, wenn mehrere potenzielle zielsprachliche Äquivalente zur Verfügung stehen und mithilfe der Evaluationsstrategien keine Version bevorzugt werden kann;

- Reduktionsstrategien, die nötig sind, wenn der Übersetzer kein zielsprachliches Äquivalent finden kann, das der ausgangssprachlichen Einheit in jeder Hinsicht entspricht.

Gegen das Modell von Krings hat Hönig folgende Kritikpunkte vorgebracht:

- Laut dem Kringsschen Modell ist dem Übersetzer immer die Problemart klar.

- Krings modelliert den Übersetzungsprozess in einer strikt festgelegten sequenziellen Struktur, die in der Praxis vernetzter und komplexer ist.

In seinem Modell versucht Hönig, diese Nachteile zu überwinden. 


\subsection{Hönigs Modell}

Das Modell von Hönig beruht auf seinen langjährigen intensiven Erfahrungen in der Übersetzungsdidaktik, in der er die LD-Methode gebraucht hat. Nach diesem Modell erfasst der Übersetzer den AT im Hinblick auf seine Übersetzung. Während des tiefen Verstehensprozesses wird dann der AT im Bewusstsein des Übersetzers projiziert und subjektiv größer betrachtet als in der realen Kommunikation, ,, denn er bindet nun mehr mentale Kapazität als dies bei der nicht übersetzungsbezogenen Rezeption eines Textes der Fall wäre " (Hönig, 1997: 55).

Der projizierte AT wird dann zum Objekt weiterer mentaler Verarbeitungsprozesse, die in zwei Verarbeitungsräumen stattfinden: dem unkontrollierten und dem kontrollierten Arbeitsraum. Der kontrollierte Arbeitsraum enthält Regeln und Richtigkeitsnachweise. Die assoziativen Prozesse im unkontrollierten Arbeitsraum führen den Übersetzer dazu, Erwartungen an den prospektiven ZT in Bezug auf seine Struktur, seinen Stil und seinen ganzen Inhalt aufzubauen.

Aus dem Zusammenspiel von projiziertem AT, prospektivem ZT und den Daten aus dem unkontrollierten Arbeitsraum beginnt der Übersetzer, seine eigene übersetzerische Makrostrategie auszuarbeiten. Diese Makrostrategie bestimmt den Bezugsrahmen für den ZT.

Mithilfe der Makrostrategie, an die alle weiteren Mikrostrategien angebunden sind, beginnt danach die Übersetzungsphase. In dieser Phase erweitert sich der unkontrollierte Arbeitsraum um die Assoziationskompetenz, die Hönig mit der angeborenen Übersetzungskompetenz gleichsetzt. Die Formulierungen des Zieltextes entstehen durch vier verschiedene Wege (Hönig, 1997: 56):

1. Als sprachlicher Reflex aus dem ersten Kontakt des projizierten $A T$ mit dem unko.Ar.

2. Als automatisierte Assoziationen aus dem unko.Ar. nach dem Erarbeiten einer Makrostrategie.

3. Aus dem ko.Ar. als Produkt einer Mikrostrategie, das vom Monitoring akzeptiert wird.

4. Als Produkt des Zusammenwirkens von unko.Ar. und ko.Ar., wobei die letzte Instanz entweder kontrollierendes Monitoring oder unkontrollierte, (intuitive oder automatisierte) Prozesse sein können. 
Der Auffassung von Hönig zufolge hängen gute Übersetzungen von der übersetzerischen Kompetenz ab, die durch das Zusammenwirken der Assoziationskompetenz mit der übersetzerischen Makrostrategie entsteht wird.

\subsection{Das Modell von Kiraly}

Wie Hönig geht Kiraly von dem Grundgedanken aus, dass gute Übersetzungen nur aus dem Zusammenspiel von Intuition und strategischer Kompetenz entstehen. Zur Entwicklung seines Modells bedient sich Kiraly der Protokolle des lauten Denkens aus einer Studie, an der neun Übersetzungsstudierende und neun professionelle Übersetzer teilgenommen haben. Unmittelbar auf die Übersetzung folgten retrospektive Interviews.

Aus den Ergebnissen seiner Studie zieht Kiraly die Schlussfolgerung, dass drei Situationskontexte das Übersetzen ausmachen:

- der Ausgangssituationskontext $\left(\mathrm{CS}_{1}\right)$, der vieles umfasst: den Autor, die Leser des Ausgangstextes, das sprachliche Material, den Einfluss des Ausgangstextes auf die Leserschaft und alles, worauf der AT referiert;

- der vom Übersetzer aufgrund seines Wissens über die Zieltextadressaten, die Situation und den Übersetzungsauftrag projizierte Zielsituationskontext $\left(\mathrm{CS}_{2}\right)$;

- der Übersetzungssituationskontext $\left(\mathrm{CS}_{3}\right)$, der als Schnittstelle zwischen den beiden anderen Kontexten funktioniert und im Selbstbild des Übersetzers manifest wird.

Das Modell Kiralys versteht sich als ein kognitives Modell des Übersetzungsprozesses. Neben den sozialen Kontexten bezieht Kiraly kognitive Komponenten des Übersetzens in sein Modell mit ein:

- Informationsquellen (Langezeitgedächtnis, Ausgangstext und externe Ressourcen);

- intuitives Verarbeitungszentrum („Relatively Uncontrolled Processing Center“);

- kontrolliertes Verarbeitungszentrum („Relatively Controlled Processing Center“).

Kiraly begreift das Gedächtnis des Übersetzers als ein Informationsverarbeitungssystem. Übersetzungen entstehen, wenn die intuitiven und kontrollierten Prozesse miteinander interagieren sowie wenn auf sprachliches und 
außersprachliches Wissen zurückgegriffen wird. Der Übersetzer speichert das gesamte Wissen in seinem Langzeitgedächtnis und bildet aufgrund der Interaktion zwischen den verschiedenen Situationskontexten und seinem Wissen die sog. Erwartungsstrukturen, die Kiraly wie folgt charakterisiert: „The expectation structure is a master plan (or a set of constrains) for a translation in progress; it is a set of potentials for a translation-to-be“ (Kiraly 1995: 102; nach Göpferich 2008: 141).

Im Unterschied zu Hönig, der zwischen dem kontrollierten und unkontrollierten Arbeitsraum unterscheidet, bevorzugt Kiraly, von einem „Relatively Controlled Processing Center“ und einem „Relatively Uncontrolled Processing Center“ zu sprechen. Aus dem relativ unkontrollierten Verarbeitungszentrum gehen tentative Übersetzungen hervor, die im relativ kontrollierten Verarbeitungszentrum bearbeitet werden. Wenn das nicht der Fall ist, entstehen Übersetzungsprobleme, $\mathrm{zu}$ deren Lösung das relativ kontrollierte Verarbeitungszentrum Strategien anwendet. Über Strategien schreibt Kiraly (1995: 105; nach Göpferich 2008: 142): „Strategies do not solve translation problems; they are merely plans carried out in an attempt to solve problems".

Aufgrund der vorgelegten Überlegungen werden die Thesen des prozessorientierten Übersetzungsansatzes wie folgt zusammengefasst (vgl. Krings 1988: 394):

1. Die mentalen Prozesse, die beim Übersetzen im Kopf des Übersetzers ablaufen, gehören unmittelbar zum Gegenstandsbereich der Übersetzungswissenschaft.

2. Die Grundlage der prozessorientierten Forschung sind die kognitiven Prozesse, die zum Produzieren des Zieltexts führen, beginnend mit der Rezeption des Ausgangstexts und den ersten Recherchierarbeiten bis $\mathrm{zu}$ den letzten Korrekturprozeduren unmittelbar vor der Abgabe der Übersetzung.

3. Der übersetzungsprozessuale Ansatz geht von empirisch-induktiven Modellen des Übersetzungsprozesses aus.

4. Der übersetzungsprozessuale Ansatz ist deskriptiv. Es beschreibt den Übersetzungsprozess der Versuchspersonen mit allen Defiziten. 
5. Die prozessorientierte Forschung stellt den Übersetzer in den Mittelpunkt des Übersetzungsprozesses und zielt auf die Erklärung des Einflusses der prozessrelevanten Variablen ab.

6. Das Hauptanliegen der prozessorientierten Forschung ist die empirische Grundlegung der Übersetzungsdidaktik.

Abschließend zum Textteil des vorliegenden Beitrags werden drei Begründungen für die Relevanz der Übersetzungsprozessforschung angeführt (vgl. Krings 2005: 344):

\section{Die wissenschaftlich-systematische Begründung}

Die Übersetzungsprozessforschung hilft den Übersetzungswissenschaftlern, die menschlichen Sprachverarbeitungsprozesse präziser zu verstehen.

\section{Die angewandt-übersetzungsdidaktische Begründung}

Die Übersetzungsprozessforschung erhellt die mentalen Prozesse, die bei Menschen mit unterschiedlicher übersetzerischer Kompetenz und unter unterschiedlichen Bedingungen während des Übersetzungsprozesses ablaufen. Das hat den Vorteil für die Übersetzungsdidaktiker und angehenden Übersetzer, dass sie ein effektives Lehren und Lernen von Übersetzungskompetenz ermöglichen können.

\section{Die standespolitische Begründung}

In Bezug auf die Berufsübersetzer trägt die Übersetzungsprozessforschung zur Stärkung des Berufsbildes und des sozialen Prestiges des Übersetzers bei, weil sie die Komplexität des Übersetzens erklärt und damit die Notwendigkeit von Professionalität in Ausbildung und Berufspraxis unterstreicht.

\section{Zusammenfassung und Ausblick}

In diesem Beitrag hat die Forscherin versucht, auf den prozessorientierten Aspekt der Übersetzungsforschung näher einzugehen. Dieser Aspekt hat sich im Hinblick auf die Kenntnisse der Kognitiven Linguistik entwickelt. Er betrachtet das Übersetzen als einen mentalen Problemlösungsprozess und zielt darauf ab, die geistige Blackbox eines Übersetzers mithilfe der kognitivistischen Paradigmen 
aufzuhellen, um Aufschlüsse über die mentalen Vorgehensweisen eines Übersetzers zu gewinnen, die beginnend mit der Rezeption des Ausgangstexts bis zur Abgabe des Zieltexts erfolgen.

Zum Schluss ist darauf hinzuweisen, dass weitere prozessuale Forschungen zu der Anwendung der dargestellten Methoden im Übersetzungsunterricht bei den ägyptischen Germanistik-Studierenden durchgeführt werden sollen. Die Ergebnisse der prozessualen Übersetzungsforschung werden vor allem den Studierenden die Komplexität des Übersetzens bewusstmachen und bei ihnen ein übersetzungsspezifisches Selbstbewusstsein aufbauen, das es ihnen ermöglicht, sich die individuellen mentalen Vorgänge während des Übersetzens steuerbar zu machen. Ergänzt werden sollen diese Erkenntnisse mit wissenschaftlichen Beiträgen zur Umsetzung der prozessualen Modelle in eine innovative Ausrichtung der Übersetzungslehre, die dem Studierenden eine aktive Rolle beim Unterrichtsablauf zuweist.

\section{Literaturverzeichnis}

Felix, S. W. / Habel, C. / Rickheit, G. (Hrsg.) (1994): Kognitive Linguistik Repräsentation und Prozesse.

Göpferich, S. (2008): Translationsprozessforschung: Stand - Methoden Perspektiven. Tübingen: Narr Francke.

Grabowski, J. (1999): Übersetzen und Dolmetschen aus kognitionspsychologischer Sicht. In: Gil, A. et al. (Hrsg.): Modelle der Translation - Grundlagen für Methodik, Bewertung, Computermodellierung. Frankfurt am Main u.a.: Lang. S. $97-121$.

Hansen, G. (2006): Erfolgreich Übersetzen - Entdecken und Beheben von Störquellen. Tübingen: Narr Francke.

Hönig, H. G. (1997): Konstruktives Übersetzen. 2., durchgesehene Auflage. Tübingen: Stauffenburg.

Krings, H. P. (1986): Was in den Köpfen von Übersetzern vorgeht - Eine empirische Untersuchung zur Struktur des Übersetzungsprozesses an fortgeschrittenen Französischlernern, Tübingen: Narr. 
Krings, H. P. (1988): Blick in die ,Black Box' - Eine Fallstudie zum Übersetzungsprozeß bei Berufsübersetzern. In: Arntz, R. (Hrsg): Textlinguistik und Fachsprache - Akten des internationalen übersetzungswissenschaftlichen AILA-Symposions Hildesheim. Hildesheim u.a.: Olms. S. $393-412$.

Krings, H. P. (2005): Wege ins Labyrinth - Fragestellungen und Methoden der Übersetzungsprozessforschung im Überblick. In: Meta 50.2. S. 342 - 358.

Orbán, W. (2008): Über die Entlehnung konstruktivistischer Lerntheorien in die Praxis der Übersetzungswissenschaft - Kooperatives Übersetzen als kommunikations- und prozessorientierte Handlungsform des Übersetzens. Trier: WVT Wissenschaftlicher Verlag Trier.

Rickheit, G. / Sichelschmidt, L. / Strohner, H. (2007): Psycholinguistik - die Wissenschaft vom sprachlichen Verhalten und Erleben. 2. Auflage. Tübingen: Stauffenburg Verlag.

Rickheit, G. / Weiss, S. / Eikmeyer, H. (2010): Kognitive Linguistik - Theorien, Modelle, Methoden. Tübingen: Narr Francke.

Risku, H. (1998): Translatorische Kompetenz - Kognitive Grundlagen des Übersetzens als Expertentätigkeit. Tübingen: Stauffenburg.

Schwarz, M. (2008): Einführung in die Kognitive Linguistik. 3., vollständig überarbeitete und erweiterte Auflage. Tübingen und Basel: Francke.

Stolze, R. (2008): Übersetzungstheorien - Eine Einführung. 5., überarbeitete und erweiterte Auflage. Tübingen: Narr: Francke.

Wilss, W. (1988): Kognition und Übersetzen - Zu Theorie und Praxis der menschlichen und maschinellen Übersetzung. Tübingen: Niemeyer.

\section{Weblinks}

http://www.uni-heidelberg.de/studium/ interesse/faecher/computerling_ba.html, am 06.12.2016. 